\title{
INCOHERENT MID-INFRARED PHOTON ECHOES WITH PARAMETRICALLY DOWNCONVERTED LIGHT
}

\author{
SANDER WOUTERSEN*, MISCHA BONN, \\ ULI EMMERICHS and HUIB J. BAKKER \\ FOM-Institute for Atomic and Molecular Physics, Kruislaan 407, \\ 1098 SJ Amsterdam, The Netherlands
}

(Received 30 April 1997)

\begin{abstract}
We present a simple method to generate mid-infrared photon echoes, using parametrically downconverted incoherent light. The photon echoes generated in this way enable the study of the dynamics of vibrations in the $1.5-4.0 \mu \mathrm{m}$ wavelength region with subpicosecond time resolution.
\end{abstract}

Keywords: Infrared; nonlinear optics; photon echoes

Recently, the first successful infrared photon-echo study on a molecular vibration was reported [1]. In this study the homogeneous dephasing of a strongly absorbing CO-stretching vibration was investigated in the time domain using the picosecond pulses of a free-electron laser. The cross sections of other vibrations, such as the chemically and biologically important $\mathrm{CH}, \mathrm{OH}$ and $\mathrm{NH}$-stretching modes, are much smaller, which might render them difficult to study in the same way. Here, we present an alternative simple method to perform mid-infrared photon-echo spectroscopy, that can also be applied to these vibrations. The method makes use of the fact that in photon-echo experiments the time resolution is not determined by the pulse duration, but rather by the coherence time $\tau_{c}$ of the pulses.

\footnotetext{
* Corresponding author.
} 
Hence, photon-echo spectroscopy can be performed with incoherent pulses that are much longer than the homogeneous dephasing time $T_{2}$ but have a shorter coherence time [2].

The experimental setup has been described in detail elsewhere [3]. The mid-infrared pulses $(20 \mathrm{ps}, 100 \mu \mathrm{J}$, wavelength tunable from 1.5 to $4.0 \mu \mathrm{m})$ are generated via parametric generation and amplification in $\mathrm{LiNbO}_{3}$. The parametric generation process starts from zero-point fluctuations of the electromagnetic field. As a result, the generated light is incoherent and the bandwidth is only limited by phase matching. The infrared pulses are split and one part is sent through a variable delay. The two beams are focused into the sample using a $100 \mathrm{~mm} \mathrm{CaF}{ }_{2}$ lens in slightly different directions $\mathbf{k}_{1}$ and $\mathbf{k}_{2}$, making an angle of $\sim 5^{\circ}$. These pulses will generate a four-wave mixing (FWM) signal in the diffracted direction $2 \mathbf{k}_{2}-\mathbf{k}_{1}$. For an inhomogeneously broadened absorption band this signal will be a photon echo.

We have applied the method to the $\mathrm{OH}$-stretching mode of a methanol solution and of a hydrogen-bonded polymer film. Figure 1 shows a scan obtained by monitoring the time-integrated intensity in the direction $2 \mathbf{k}_{2}-\mathbf{k}_{1}$ as a function of delay, at a wavelength of $3.0 \mu \mathrm{m}$, for a $2.2 \mathrm{M}$ solution of methanol in bromoform at room temperature. The signal consists of a coherence spike with a width of approximately 1 ps superposed on a broad background with a width of approximately 20 ps. For small delays, the two incoherent pulses $\mathbf{k}_{1}$ and $\mathbf{k}_{2}$ are strongly correlated and generate a strong FWM signal. This signal forms the coherence spike and its decay with increasing delay reflects the homogeneous dephasing if $T_{2}>\tau_{c}$ [4]. In Figure 1, the coherence spike is symmetric around delay zero. This indicates that under these circumstances the homogeneous dephasing time $T_{2}$ is much shorter than $\tau_{c}$, and that the width of the coherence spike is determined only by $\tau_{c}$. Note that the coherence time is about 20 times smaller than the pulse duration, demonstrating clearly that the pulses are incoherent.

The polymer sample consisted of poly(vinylbutyral). Figure 2 presents delay scans of the coherence spike generated by exciting the $\mathrm{OH}$-stretch mode in this polymer at an excitation wavelength of $2.9 \mu \mathrm{m}$, at room temperature and at $15 \mathrm{~K}$ (the curves represent a calculation). At room temperature the signal is symmetric around delay zero, implying that $T_{2} \ll \tau_{c}$, as in Figure 1. A least-squares fit of the calculation to the room temperature data yields a value for $\tau_{c}$. 


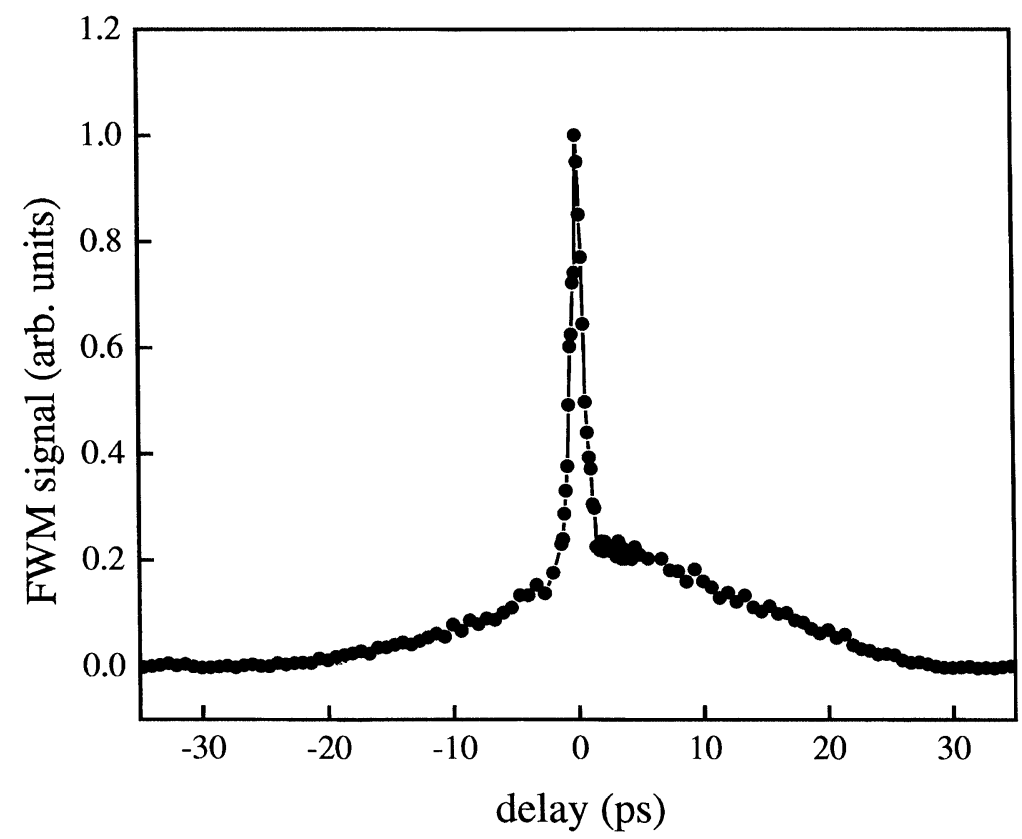

FIGURE 1 Integrated four-wave mixing intensity as a function of delay between $\mathbf{k}_{1}$ and $\mathbf{k}_{2}$, at an excitation wavelength of $3.0 \mu \mathrm{m}$ in a $100 \mu \mathrm{m}$ thick sample consisting of a $2.2 \mathrm{~mol} \mathrm{dm}^{-3}$ solution of methanol in bromoform at room temperature. The signal consists of a symmetric coherence spike with a width of $\sim 1$ ps superposed on a broad background with a width of $\sim 20 \mathrm{ps}$.

From a least-squares fit to the $15 \mathrm{~K}$ data, in which the same $\tau_{c}$ was used, a value for $T_{2}$ of $2.8 \pm 0.3 \mathrm{ps}$ was obtained. This value corresponds to a homogeneous linewidth of $\sim 4 \mathrm{~cm}^{-1}$, which is nearly two orders of magnitude smaller than the absorption linewidth of $\sim 170 \mathrm{~cm}^{-1}$ [5].

There are two important advantages to the method presented here as compared to mid-infrared photon-echo spectroscopy using a freeelectron laser. First, the fact that the coherence time is so much shorter than the pulse duration renders this experiment equivalent to an accumulated photon echo [6], provided the excited state lifetime $T_{1}$ is longer than the coherence time. The accumulation of the grating amplitude during the incoherent pulse results in an echo signal which is orders of magnitude larger than could be generated with equally intense coherent pulses of length $\tau_{c}$. This enables the study of 


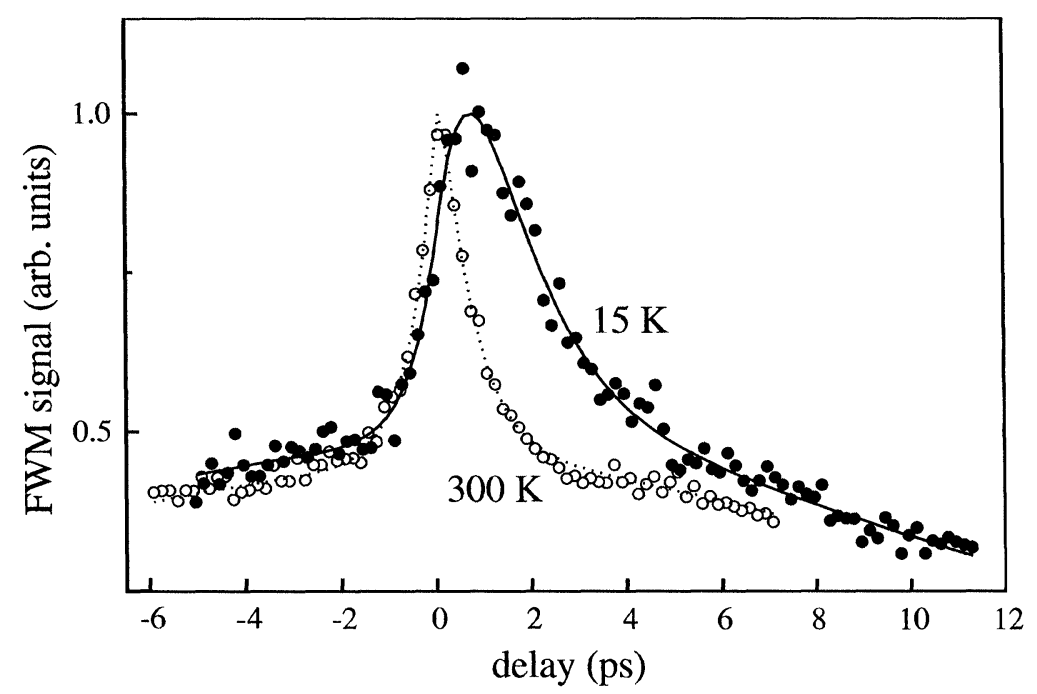

FIGURE 2 Integrated four-wave mixing intensity as a function of delay between $\mathbf{k}_{1}$ and $\mathbf{k}_{2}$, at an excitation wavelength of $2.9 \mu \mathrm{m}$ in poly(vinylbutyral) at room temperature and $15 \mathrm{~K}$. The sample consisted of a $50 \mu \mathrm{m}$ layer of poly(vinylbutyral) deposited on a sapphire plate, following the procedure of Ref. [5]. The curves have been calculated using the model described in Ref. [4].

vibrations with small absorption cross sections, notably the $\mathrm{OH}, \mathrm{CH}$ and $\mathrm{NH}$-stretching modes. Second, the setup required to generated the incoherent mid-infrared pulses is compact and can easily be tuned over a large wavelength region.

\section{References}

[1] Zimdars, D., Tokmakoff, A., Chen, S., Greenfield, S. R. and Fayer, M. D. (1993). Phys. Rev. Lett., 70, 2718.

[2] Asaka, S., Nakatsuka, H., Fujiwara, M. and Matsuoka, M. (1984). Phys. Rev. A, 29, 2286.

[3] Woutersen, S., Bonn, M., Brugmans, M. J. P., Emmerichs, U. and Bakker, H. J. (1996). Opt. Lett., 21, 1579.

[4] Morita, N. and Yajima, T. (1984). Phys. Rev. A, 30, 2525.

[5] Graener, H., Ye, T. Q. and Laubereau, A. (1990). Phys. Rev. B, 41, 2597.

[6] Bai, Y. S. and Fayer, M. D. (1988). Chem. Phys., 128, 135. 